\title{
Antidote-Controlled Platelet Inhibition Targeting von Willebrand Factor with Aptamers
}

\author{
S. ONEY, ${ }^{1-3}$ S.M. NIMJEE,,${ }^{1,2,4}$ J. LAYZER, ${ }^{1,2}$ N. QUE-GEWIRTH,${ }^{1,2}$ D. GINSBURG, ${ }^{6}$ \\ R.C. BECKER, ${ }^{1,5}$ G. AREPALLY, ${ }^{5}$ and B.A. SULLENGER ${ }^{1-3}$
}

\begin{abstract}
Thrombus formation is initiated by platelets and leads to cardiovascular, cerebrovascular, and peripheral vascular disease, the leading causes of morbidity and mortality in the Western world. A number of antiplatelet drugs have improved clinical outcomes for thrombosis patients. However, their expanded use, especially in surgery, is limited by hemorrhage. Here, we describe an antiplatelet agent that can have its activity controlled by a matched antidote. We demonstrate that an RNA aptamer targeting von Willebrand factor (VWF) can potently inhibit VWF-mediated platelet adhesion and aggregation. By targeting this important adhesion step, we show that the aptamer molecule can inhibit platelet aggregation in PFA-100 and ristocetin-induced platelet aggregation assays. Furthermore, we show that a rationally designed antidote molecule can reverse the effects of the aptamer molecule, restoring platelet function quickly and effectively over a clinically relevant period. This aptamer-antidote pair represents a reversible antiplatelet agent inhibiting a platelet specific pathway. Furthermore, it is an important step towards creating safer drugs in clinics through the utilization of an antidote molecule.
\end{abstract}

\section{INTRODUCTION}

$\mathbf{P}$ LATELETS are anuclear, disk-shaped cells that play a key role in hemostasis and thrombosis. They survey the blood vessels, arrest bleeding around areas of damage and are a critical factor in maintaining hemostasis. However, they can also lead to thrombosis, which is the leading cause of morbidity and mortality in the Western world (Jackson and Schoenwaelder, 2003; Thom et al., 2006).

In the last 20 years, a number of antiplatelet drugs have emerged as more information about platelet biology revealed novel targets for therapeutic inhibition. Although these agents have had a major impact on reducing the complications of cardiovascular disease, their therapeutic effect is incomplete for many high-risk patients (Bhatt and Topol, 2003; Jackson and Schoenwaelder, 2003). Many of these drugs have been directed at a specific pathway in platelet activation (i.e., aspirin and clopidogrel). A fundamental problem with targeting a specific pathway is that platelet aggregation can still occur via alternate pathways, leading to a relatively mild antithrombotic effect. It is estimated that approximately $8 \%-18 \%$ of patients treated with aspirin experience recurrent vascular events after 2 years (Mason et al., 2005). Although platelet receptor glycoprotein IIIb-IIIa (GP IIIb-IIIa) inhibitors overcome this problem by targeting the final common pathway in platelet activation, their therapeutic efficacy is limited by bleeding at peak concentrations and subtherapeutic activity at lower doses (Wu et al., 2002a). Furthermore, none of the current antiplatelet therapies prevent the initiating step for thrombus formation:

${ }^{1}$ Duke Translational Research Institute, ${ }^{2}$ Department of Surgery, ${ }^{3}$ University Program in Genetics and Genomics, ${ }^{4}$ Division of Neurosurgery, ${ }^{5}$ Department of Medicine, Duke University Medical Center, Durham, North Carolina.

${ }^{6}$ University of Michigan, Ann Arbor, Michigan. 
platelet adhesion (Bhatt and Topol, 2003; Jackson and Schoenwaelder, 2003). The current classes of antiplatelet agents (aspirin, thienopyridines, and the glycoprotein IIb/IIIa inhibitors) target metabolic or aggregatory properties of platelets, but do little to interfere with plateletvessel wall interactions; it is in these interactions that circulating von Willebrand factor (VWF) and subendothelial matrix proteins play an important role.

VWF is a multimeric plasma glycoprotein that is critical for primary hemostasis. It plays a direct role in allowing platelet attachment to sites of vascular injury through their GP Ib-IX-V receptors and aids in platelet plug formation by enabling platelet-platelet interactions through GP IIb-IIIa (Ruggeri et al., 1999; Ruggeri 2003). Qualitative or quantitative deficiencies of VWF can lead to von Willebrand's disease (VWD), in which patients present with a mild to severe bleeding phenotype (Sadler 2005a, 2005b). Numerous studies have shown that inhibiting the VWF-GP Ib-IX-V interaction using antibodies can decrease arterial thrombosis in animal models, but these approaches have serious shortcomings that impede their clinical development (Miller et al., 1991; Cadroy et al., 1994; Chang et al., 1998; Yamamoto et al., 1998; Cauwenberghs et al., 2000; Wu et al., 2002; Bhatt and Topol, 2003; Jackson and Schoenwaelder, 2003). None of these molecules are reversible, and at doses that can effectively inhibit VWF activity, they transiently engender a VWD-disease state that can cause severe bleeding, thus undermining patient safety (Cadroy et al., 1994; Yamamoto et al., 1998; Wu et al., 2002). The fact that VWD results in patients with bleeding disorders who may require medical therapy certainly validates VWF as an attractive target for platelet inhibition, but one that would require stringent control so as to provide clinical benefit without the risk of hemorrhage.

The case for antidote control of antiplatelet agents is no clearer than in the surgical arena where minimizing comorbidities improves outcomes. Many patients taking aspirin and clopidorel are advised to stop taking their medication 7-10 days prior to surgery so as to prevent bleeding complications. This places the patient at risk for thrombosis and creates a therapeutic dilemma for surgeons. Moreover, a number of studies have shown that coupling antiplatelet agents with anticoagulant therapies can improve patient outcomes but carries a significant risk of hemorrhage (Huynh et al., 2001). It is this increased risk of major bleeding that lowers the clinical benefit of such combination therapies. An antidote-controlled antiplatelet drug would provide the flexibility of protecting the patient from thrombotic complications and allow for rapid reversal of the drug's activity in a lifethreatening hemorrhage situation or in order to perform an invasive procedure.

To generate a safer, antidote-controllable VWF inhibitor, we decided to exploit the properties of nucleic acid ligands termed aptamers. Aptamers are single-stranded nucleic acid molecules that can directly inhibit protein function by binding to their targets with high affinity and specificity (Nimjee et al., 2005a, 2005b). To isolate RNA aptamers against VWF, we performed a modified version of SELEX (systematic evolution of ligands by exponential enrichment) termed convergent SELEX. These aptamers bind to VWF with high affinity $\left(\mathrm{K}_{\mathrm{d}}<20 \mathrm{nM}\right)$ and inhibit platelet aggregation in platelet function analyzer (PFA-100) and ristocetin-induced platelet aggregation (RIPA) assays. Moreover, we have rationally designed an antidote molecule that can quickly reverse above said aptamers' function. This antidote molecule can give physicians better control in clinics, enhancing the aptamers' safety profile.

\section{MATERIALS AND METHODS}

\section{Generation of aptamers}

Convergent SELEX. The sequence of the starting RNA combinatorial library was 5'-GGGAGGACGATGCGG$\mathrm{N}_{40}$-CAGACGACTCGCTGAGGATCC-3', where $\mathrm{N}_{40}$ represents 40 completely random nucleotides. $2^{\prime} \mathrm{F}$ cytidine triphosphate and $2^{\prime} \mathrm{F}$ uridine triphosphate (Trilink Biotechnologies, San Diego, CA) were incorporated into the RNA libraries by in vitro transcription in order to confer nuclease resistance. The selection was carried out in selection buffer E (20 mM HEPES, pH 7.4, $50 \mathrm{mM}$ $\mathrm{NaCl}, 2 \mathrm{mM} \mathrm{CaCl}$, and $0.1 \%$ bovine serum albumin [BSA]) at $37^{\circ} \mathrm{C}$ until round P5V2 and then continued in selection buffer $\mathrm{F}$ (20 mM HEPES, pH 7.4, $150 \mathrm{mM}$ $\mathrm{NaCl}, 2 \mathrm{mM} \mathrm{CaCl}$, and $0.1 \% \mathrm{BSA}$ ). RNA-VWF complexes were separated from unbound RNA by passing them over a nitrocellulose filter (BA 85, Whatman Inc., Florham Park, NJ).

Five rounds of SELEX were performed on the plasma proteome followed by four rounds of convergent SELEX as described by Layzer et al. (2007). Briefly, the starting aptamer library (Sel2) was incubated with diluted normal human plasma at $37^{\circ} \mathrm{C}$ for 15 minutes in selection buffer E. Yeast tRNA was used to inhibit nonspecific binding of the aptamer library to the plasma proteome. Bound RNA aptamers were separated from unbound aptamers using a nitrocellulose filter. After round 5 of plasma SELEX, convergent SELEX using VWF was performed for 4 rounds ( 2 rounds in selection buffer $\mathrm{E}$ followed by 2 rounds in selection buffer $\mathrm{F}$ ).

\section{Antidote oligonucleotides}

Antidote oligonucleotides were synthesized and purified by Dharmacon Research, Inc. (Lafayette, CO). 2'-Omethyl purines and pyrimidines were incorporated into the antidote oligonucleotides. 


\section{Binding assays}

Affinity constants ( $\mathrm{K}_{\mathrm{d}}$ values) were determined using double-filter nitrocellulose filter binding assays (Rusconi et al., 2000). All binding studies were performed in either binding buffer $\mathrm{E}$ (20 mM HEPES, pH 7.4, $50 \mathrm{mM} \mathrm{NaCl}$, $2 \mathrm{mM} \mathrm{CaCl}$, and $0.1 \% \mathrm{BSA}$ ) or binding buffer $\mathrm{F}(20$ mM HEPES, $\mathrm{pH} 7.4,150 \mathrm{mM} \mathrm{NaCl}, 2 \mathrm{mM} \mathrm{CaCl}$, and $0.1 \% \mathrm{BSA}$ ) at $37^{\circ} \mathrm{C}$. Human purified VWF (factor VIII free) was purchased from Haematologic Technologies Inc. (Essex Junction, VT) and used in the double-filter nitrocellulose filter binding assay to determine the $\mathrm{K}_{\mathrm{d}}$ of every other round and individual clones. VWF SPI and VWF SPIII domains were kindly provided by Dr. J. Evan Sadler (Washington University, St. Louis, MO). Briefly, RNA were dephosphorylated using bacterial alkaline phosphatase (Gibco BRL, Gaithersberg, MD) and end-labeled at the $5^{\prime}$ end with $\mathrm{T} 4$ polynucleotide kinase (New England Biolabs, Beverly, MA) and $\left[\gamma^{32} \mathrm{P}\right]$ ATP (Amersham Pharmacia Biotech, Piscataway, NJ; Fitzwater and Polisky, 1996). Direct binding was performed by incubating ${ }^{32} \mathrm{P}-\mathrm{RNA}$ with VWF in physiologic buffer plus 1 $\mathrm{mg} / \mathrm{mL}$ bovine serum albumin (BSA) at $37^{\circ} \mathrm{C}$ for $5 \mathrm{~min}$ utes. The fraction of the nucleic acid-protein complex that bound to the nitrocellulose membrane was quantified with a phosphoimager (Molecular Dynamics, Sunnyvale, CA). Nonspecific binding of the radiolabeled nucleic acid was subtracted out of the binding such that only specific binding remained (Wong and Lohman, 1993).

\section{Platelet function analysis}

PFA-100. The Platelet Function Analyzer, PFA-100 (Dade Behring, Deerfield, IL), measures platelet function in terms of clot formation time. In this assay, we utilized collagen/ADP cartridges to activate the platelets and measure the amount of time taken to form a clot in anticoagulated whole blood (Harrison, 2005). Briefly, 840 $\mu \mathrm{L}$ of whole blood was mixed with aptamer in platelet binding buffer (150 mM NaCl; $20 \mathrm{mM}$ Hepes pH: 7.4; 5 $\mathrm{mM} \mathrm{KCl} ; 1 \mathrm{mM} \mathrm{MgCl}{ }_{2}$ and $1 \mathrm{mM} \mathrm{CaCl}{ }_{2}$ ) and incubated for 5 minutes at room temperature. This mixture was then added to a collagen/ADP cartridge and tested for its closing time. The maximum closing time of the PFA-100 is 300 seconds. Antidote activity of the aptamer was measured by mixing whole blood with aptamer, incubating for 5 minutes followed by addition of antidote or buffer, and testing the mixture in the PFA-100.

Platelet aggregometry. A Chrono-log Whole Blood Lumi Ionized Aggregometer (Chrono-log, Haverton, PA) was used to provide a measurement of platelet aggregation in platelet-rich plasma (PRP). Briefly, PRP was isolated from whole blood collected in 3.2\% buffered trisodium citrate tubes (BD Vacutainer Systems, Franklin Lakes, NJ); aptamer was added and incubated with the
PRP for 5 minutes before testing. After calibrating the instrument, $5 \mu \mathrm{L}$ of agonist was added and transmission was measured for 10 minutes.

Ristocetin-induced platelet aggregation. Ristocetin-induced platelet aggregation (RIPA) was performed using platelet-rich plasma (PRP) from healthy volunteers. Clone VWF R9.3 or VWF R9.14 was mixed with $400 \mu \mathrm{L}$ of PRP in a flat-bottom glass tube; ristocetin (Helena Laboratories, Beaumont, TX) was added to a final concentration of $1.25 \mathrm{mg} / \mathrm{mL}$. The PRP was stirred using a steel stir bar at $37^{\circ} \mathrm{C}$ and turbidity was monitored as percent light transmitted for 10 minutes.

Collagen-induced platelet aggregation. Collagen-induced platelet aggregation (CIPA) was performed using PRP from healthy volunteers. Clone VWF R9.3 or VWF R9.14 was mixed with $400 \mu \mathrm{L}$ of PRP in a flat-bottom glass tube and collagen was added to a final concentration of $2 \mu \mathrm{g} / \mathrm{mL}$. The PRP was stirred using a steel stir bar at $37^{\circ} \mathrm{C}$ and turbidity was monitored as percent light transmitted for 10 minutes.

ADP-induced platelet aggregation. ADP-induced platelet aggregation (AIPA) was performed using PRP from healthy volunteers. Clone VWF R9.3 or VWF R9.14 was mixed with $400 \mu \mathrm{L}$ of PRP in a flat-bottom glass tube and ADP was added to a final concentration of $10 \mu \mathrm{M}$. The PRP was stirred using a steel stir bar at $37^{\circ} \mathrm{C}$ and turbidity was monitored as percent light transmitted for 6 minutes.

Thrombin-induced platelet aggregation. Thrombin-induced platelet aggregation (TIPA) was performed using PRP from healthy volunteers and SFLLRN peptide. Clone VWF R9.3 or VWF R9.14 was mixed with $400 \mu \mathrm{L}$ of PRP in a flat-bottom glass tube and SFLLRN was added to a final concentration of $2 \mathrm{nM}$. The PRP was stirred using a steel stir bar at $37^{\circ} \mathrm{C}$ and turbidity was monitored as percent light transmitted for 6 minutes.

\section{RESULTS}

Five rounds of SELEX followed by four rounds of convergent SELEX yielded aptamers that bind to $V W F$ with high affinity.

To isolate RNA aptamers against VWF, we performed a modified version of SELEX. First, an RNA library containing 2 '-flouropyrimidines was incubated with total plasma proteins; the RNA ligands that bound to this proteome were recovered. Four additional rounds of SELEX were performed against the plasma proteome to generate a focused library that was highly enriched for RNA ligands that bound plasma proteins. Next, convergent SE- 
LEX (Layzer, 2007) was performed to isolate those RNA aptamers from the focused library that specifically bound VWF. Such convergent SELEX progressed rapidly; the affinity of the round 4 pool of aptamers had a $\mathrm{K}_{\mathrm{d}}$ of $4.5 \mathrm{nM}$ for VWF (Fig. 1A). Next, the identity of the individual aptamers was determined by cloning and sequencing. As shown in Table 1, two sequences dominated following round 4 of convergent SELEX against VWF (clones 9.3 and 9.4). These dominant clones, along with two less abundant clones (9.18 and 9.14), were chosen for further evaluation. To characterize the binding of these aptamers to VWF, we performed nitrocellulose filter binding assays. As shown in Table 1 and Figure 1B, three of the four clones $(9.3,9.4$, and 9.14) bound to VWF with high affinity $\left(\mathrm{K}_{\mathrm{d}}<20 \mathrm{nM}\right)$ (Fig. 1B). Thus, by performing 5 rounds of SELEX on the plasma proteome followed by 4 rounds

A

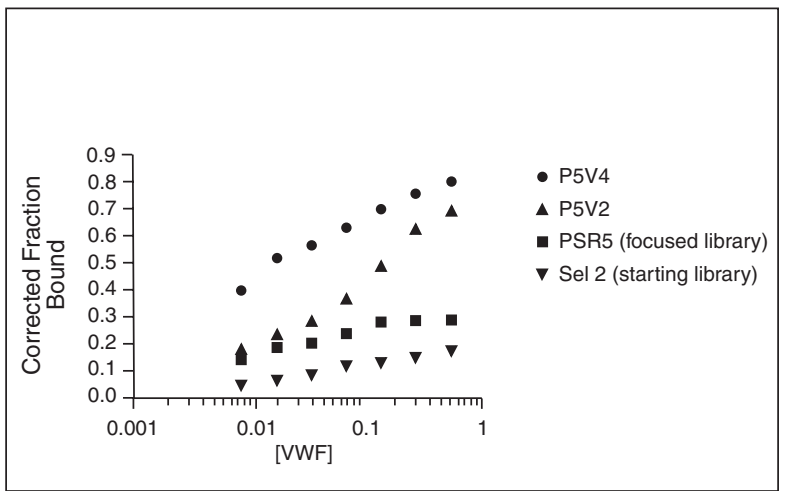

C

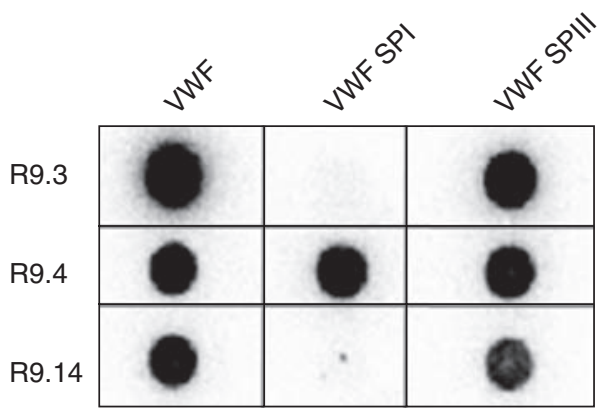

of convergent SELEX against VWF using the plasma proteome-focused library, we identified aptamers that bound to VWF with high affinity.

\section{Clones VWF R9.3 and VWF R9.4 bind to the} VWF SPIII domain but not to the VWF SPI domain; clone VWF R9.4 binds to both the VWF SPI and SPIII domains

To determine the specific binding domains of selected aptamer clones on VWF, we performed studies using VWF SPI and VWF SPIII domains. SPI and SPIII are V8 protease fragments of VWF from the N-terminus of the protein. SPIII is 1365 residues in length (aa 1-1365) containing domains from $\mathrm{D}^{\prime}$ mid-way through $\mathrm{D} 4$, including the A1 domain. SPI represents the C-terminal

B

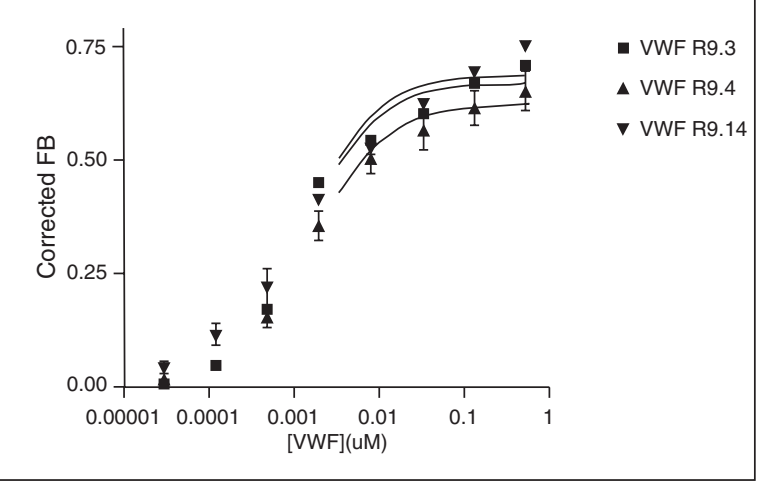

D

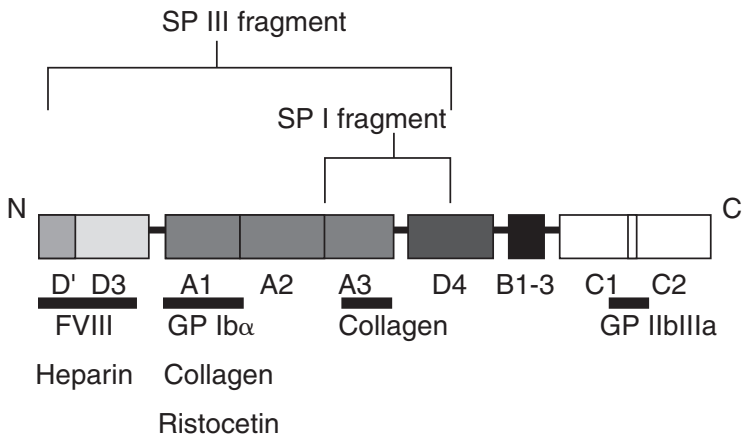

FIG. 1. Convergent systematic evolution of ligands by exponential enrichment (SELEX) yielded aptamers that bind to von Willebrand factor (VWF) with high affinity. A: Progress of the convergent SELEX was followed using a nitrocellulose filter binding assay. Inverted triangles ( $\boldsymbol{\nabla})$ represent the starting RNA library (Sel2). Squares ( $\boldsymbol{\square}$ ) represent the plasma focused library. Triangles $(\mathbf{\Delta})$ represent convergent SELEX round 2 and circles $(\mathbf{O})$ represent convergent SELEX round 4. The X-axis represents VWF concentration and the Y-axis represents the fraction of RNA bound to the protein. B: Binding affinities of VWF aptamers R9.3, R9.4 and R9.14 were determined using a nitrocellulose filter binding assay. Squares $(\boldsymbol{\square})$ represent R9.3, triangles $(\mathbf{\Delta})$ represent R9.4 and inverted triangles $(\boldsymbol{\nabla})$ represent R9.14. Each data point was done in triplicate; error bars represent the standard error of the mean (SEM) of the data. C: Binding of aptamers to VWF, VWF SPI, and VWF SPIII fragments was determined using a nitrocellulose filter binding assay. Aptamers R9.3 and R9.14 bind to both full length VWF and the VWF SPIII fragment but not to the VWF SPI fragment. Aptamer R9.4 binds to full-length VWF, the VWF SPIII and the VWF SPI fragment. D: Cartoon depicting VWF, its subunits and SPI and SPIII fragments. Binding sites for various ligands are indicated. 
Table 1. P5V4 Aptamer Sequences

\begin{tabular}{|c|c|c|c|}
\hline Clone ID & Variable region sequence & $\begin{array}{c}\text { Frequency } \\
(\%)\end{array}$ & Kd \\
\hline VWF R9.3 & 5'-ATCGCGCTCTCCTGCTTAAGCAGCTATCAAATAGCCCACT-3' & 39 & $1.2 \mathrm{nM}$ \\
\hline VWF R9.4 & 5'-TATAGACCACAGCCTGAGATTAACCACCAACCCAGGACT-3' & 36 & $1.9 \mathrm{nM}$ \\
\hline VWF R9.18 & 5'-TGCTCCTTGGCCTTAGCCCTGGAACCATCAATCCTCTTCG-3' & 3 & $278 \mathrm{nM}$ \\
\hline VWF R9.14 & 5'-TGGACGAACTGCCCTCAGCTACTTTCATGTTGCTGACGCA-3' & 1 & $12 \mathrm{nM}$ \\
\hline VWF R9.90 & 5'-ACGNGTANACCTGCTACAATANCAGCCTAAATGGCCACT-3' & 1 & $\mathrm{~N} / \mathrm{D}$ \\
\hline VWF R9.66 & 5'-ATCCCTGCCAAACATACTTTCGCTTTGGCTAGGACTCCCT-3' & 3 & N/D \\
\hline VWF R9.37 & 5'-GCACCCCCTCGACAACGACCCTGTGCCCCTCGATCGACCA-3' & 2 & N/D \\
\hline VWF R9.54 & 5'-CCCATTACGGCTT-CCTTGTATTCTTGGACAAGCCGCGACT-3' & 2 & N/D \\
\hline VWF R9.35 & 5'-ACCCTTGACAACAACCCТTCCTCACCAACCССТСССАAC-3' & 1 & N/D \\
\hline VWF R9.81 & 5'-ATACCCTCGACAACGACCCTATTCGCATGACACCTCTGTG-3' & 1 & N/D \\
\hline VWF R9.33 & 5'-ATGAATCCTCCTGTCGAACAACAGCTGTTTCAGCCCAACT-3' & 1 & N/D \\
\hline VWF R9.93 & 5'-GACCGACTGATTCGCACCAGACCACGACGTTATGGCCCAA-3' & 1 & N/D \\
\hline VWF R9.74 & 5'-GTCGACTTAGCCCCGTGCTCGGCGCTTCACAGTCGACTAT-3' & 1 & N/D \\
\hline VWF R9.41 & 5'-CGAGATCACACTGCCCCAATAGCCACTGAACTAGCGCGCA-3' & 1 & N/D \\
\hline VWF R9.46 & 5'-ACCATTCGCGAGCACAACGCTTTGTACTCAACACTCCACG-3' & 1 & $\mathrm{~N} / \mathrm{D}$ \\
\hline VWF R9.49 & 5'-ACCGTTCAGAAATGACCCCACGCACATCCATCCCTGAGCT-3' & 1 & N/D \\
\hline VWF R9.97 & 5'-ACGTGATCCTCGGACCCAGCATTGCATTATATGCGCCCCT & & N/D \\
\hline VWF R9.95 & 5'-ACTCTCAGCCCATGTGCCTCAACCAAGGCACGGCTTGCTC-3' & 1 & N/D \\
\hline VWF R9.62 & 5'-CACCCTTCACCCGAACCCTGCCC-ACG-ACCCCACACCCCGC-3' & 1 & N/D \\
\hline VWF R9.57 & 5'-ATGACCAGCCCCTCGACAACGACCCTGCTGGCTCAACCGTT-3' & 1 & N/D \\
\hline VWF R9.118 & 5'-GACCGCCGCNNCCGACCCNAGNNNTGCTGTGTNCGCTCCGCC-3' & 1 & N/D \\
\hline
\end{tabular}

$\mathrm{ND}$, not determined.

455 residues of SPIII and contains mainly domain A3 and a part of domain D4 (Fig. 1D).

Clones VWF R9.3 and VWF R9.14 bound to the SPIII fragment but not to the SPI fragment (Fig. 1C and 1D). These results suggest that these aptamers bind proximal to the positively charged A1 domain of VWF. The A1 domain is mainly involved in platelet aggregation since it makes contact with the GP Ib $\alpha$ subunit of platelet receptor GP Ib-IX-V. Clone VWF R9.4 bound to both SPI and SPIII domains, mapping its binding proximal to the VWF A3 domain (Fig. 1C).

Clones VWF R9.3 and VWF R9.14 but not VWF R9.4 inhibited platelet function measured by PFA-100

To determine whether the isolated aptamers had any effect on platelet activity, they were evaluated for their ability to limit platelet-induced clot formation in a PFA100 assay. The PFA-100 instrument uses small membranes coated with collagen/ADP or collagen/epinephrine to screen for the presence of platelet function defects. As shown in Figure 2A, VWF aptamers R9.3 and R9.14 inhibited platelet dependent clot formation completely in the PFA-100 assay (closing time $>300 \mathrm{sec}-$ onds) at a concentration of $1 \mu \mathrm{M}$. In contrast, VWF ap- tamer R9.4, while having a $\mathrm{K}_{\mathrm{d}}$ similar to R9.3 and R9.14, had no activity (Fig. 2A). Next, to determine the minimum effective dose of VWF aptamer R9.3 and VWF aptamer R9.14, we performed a dose titration study. As shown in Figure 2B, both aptamers completely inhibited platelet function (CT $>300$ seconds) at concentrations greater than $40 \mathrm{nM}$ in normal whole blood in the PFA100 assay (Fig. 2B). Thus, at concentrations above 40 $\mathrm{nM}$, these two aptamers inhibit platelet function to the level seen in patients with severe VWD.

Clones VWF R9.3 and VWF R9.14 inhibited platelet aggregation as measured by RIPA but not by CIPA, AIPA, and TIPA

To confirm these findings and to determine the specificity of the VWF aptamers, we performed platelet aggregation studies. First, we investigated the effects of VWF aptamers R9.3 and R9.14 in a RIPA assay to determine if the aptamers inhibit platelet function by blocking VWF's ability to interact with GP Ib-IX-V. Ristocetin was used as a VWF antagonist because it binds specifically to VWF in PRP and assists in VWF-mediated platelet activation/aggregation through the GP Ib-IX-V receptor. We also evaluated other antagonists (collagen, ADP, and thrombin) that activate platelets through pathways that 
are not dependent on the VWF-GP Ib-IX-V interaction to determine if the aptamers had any inhibitory effect on these additional activation pathways. As shown in Figure 2C, VWF aptamers R9.3 and R9.14 completely inhibited RIPA (at a concentration of $250 \mathrm{nM}$ ), illustrating that the aptamers can potently inhibit the VWF-GP Ib-IX-V interaction. In contrast, the aptamers had no effect in collagen, ADP or thrombin induced platelet aggregation (Fig.

\section{A}

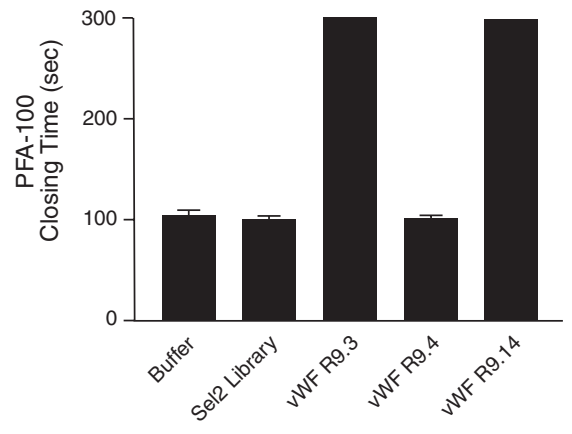

B

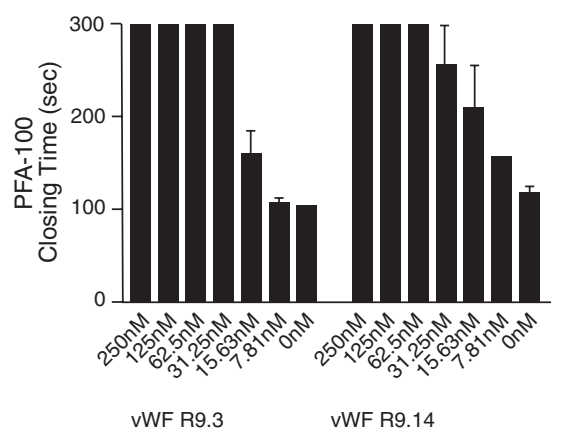

C

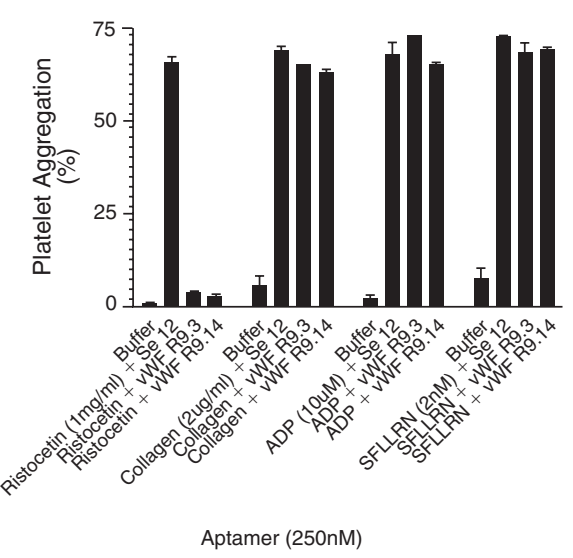

2C). Thus, VWF aptamers R9.3 and R9.14 inhibit platelet function by specifically blocking VWF-GP IbIX-V-mediated platelet activation and aggregation.

\section{Antidote oligonucleotide 6 (AO6) can reverse} VWF R9.14 binding to VWF to background levels

Six different antidote oligonucleotides (AO1-6) were designed to bind to VWF aptamer R9.14 through Watson-Crick base pairing rules (Fig. 3A). This strategy has been successfully employed in our laboratory to design an antidote to control the activity of an aptamer to factor IXa (Rusconi et al., 2002, 2004; Nimjee et al., 2006). To determine if the antidote oligonucleotides could inhibit aptamer binding to VWF, we evaluated them in a nitrocellulose filter binding assay. As shown in Figure 3B, the most effective antidote for VWF aptamer R9.14 is AO6. This antidote can reverse VWF aptamer R9.14's ability to bind VWF to background levels (Fig. 3B).

\section{AO6 can reverse the effects of VWF R9.14 completely in a PFA-100 assay}

Since AO6 can reverse VWF aptamer 9.14 binding to VWF, we wanted to next determine if the antidote could also reverse the aptamer's activity in a whole blood clinical laboratory assay. To that end, we tested the ability of AO6 to inhibit VWF aptamer 9.14 in a PFA-100 assay. As shown in Figure 4A, the antidote can reverse the activity of the aptamer in a dose-dependent manner. Moreover, the antidote is able to completely reverse the antiplatelet effects of the VWF aptamer R9.14 at a 40-fold excess of aptamer concentration. In contrast, a scrambled version of the antidote oligonucleotide (Scr AO6) had no effect on aptamer activity (Fig. 4A). Thus, antidote AO6 is able to restore platelet function in a whole blood assay back to normal levels, even in the presence of enough VWF aptamer $9.14(40 \mathrm{nM})$ to impede platelet function to an extent consistent with VWD.

FIG. 2. Von Willebrand factor (VWF) aptamers R9.3 and R9.14 inhibit platelet aggregation by blocking the VWF-GP Ib-IX-V interaction. A: The function of VWF aptamers R9.3, R9.4, and R9.14 was measured at a $1 \mu \mathrm{M}$ concentration in a PFA-100 assay. Platelet buffer and starting aptamer library (Sel2) were used as negative controls. Error bars represent the range of data. Each data point was done in triplicate. B: Varying concentrations of VWF aptamers R9.3 and R9.14 were added to normal whole blood; closing times were measured in a PFA-100 assay using collagen/ADP cartridges. Error bars represent the range of data. Each data point was done in triplicate. C: VWF aptamers R9.3 and VWF R9.14 were tested in ristocetin, collagen, ADP, and thrombin (SFLLRN) induced platelet aggregation. Filled bars represent percent aggregation in normal platelet rich plasma. Error bars represent the range of data; each data point was done in triplicate. 


\section{A}

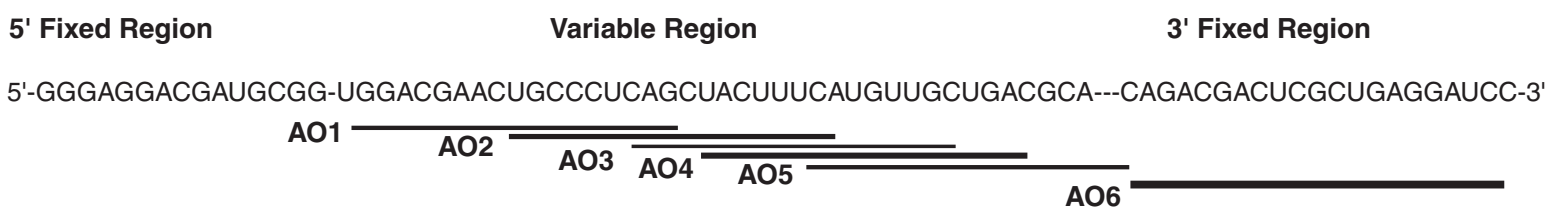

B

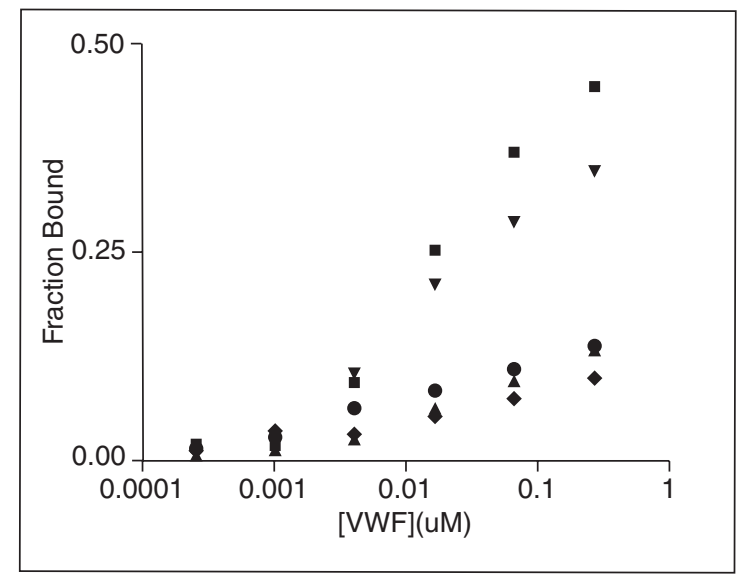

AO6 can quickly reverse the effects of VWF R9.14 for a sustained period of time in a PFA100 assay

For such an antidote to be useful clinically, the antidote should be able to act quickly and for a prolonged period of time. To determine how rapidly AO6 could reverse the aptamer and how long such reversal is sustained, we performed a time course assay using the PFA-100. As shown in Figure 4B, AO6 can rapidly reverse the effects of VWF aptamer R9.14 in less than 2 minutes. Moreover, once the antiplatelet activity is reversed, the antidote maintained its ability to sustainably inhibit the aptamer for greater than 4 hours (Fig. 4C). We could not test AO activity for more than 4 hours due to platelet degradation over such time. These results demonstrate that AO6 can rapidly and durably reverse the effects of VWF aptamer R9.14.

\section{DISCUSSION}

Aptamers are single-stranded nucleic acid molecules that can directly inhibit protein function by binding to their target with high affinity and specificity. To date, a number of proteins involved in coagulation have been targeted by aptamers, successfully yielding anticoagulant molecules with therapeutic potential (Rusconi et al., 2000a, 2002b; Becker et al., 2005; Nimjee et al., 2005). Aptamers represent an at-
FIG. 3. Antidote oligonucleotides to R9.14 can inhibit aptamer binding to von Willebrand factor (VWF). A: Cartoon depicting the antidote design to aptamer VWF R9.14. Black bars depict the positions of sequence complementarities. B: Reversal of aptamer VWF R9.14 binding to VWF was accomplished by antidote oligonucleotide 6 (AO6) (triangles) but not by AO5 (inverted triangles). AO6 and AO5 together (diamonds) also inhibit aptamer binding to VWF. The starting library (Sel2; circles) was used as a control.

tractive class of therapeutic compounds for numerous reasons. They are relatively small $(8 \mathrm{kd}$ to $15 \mathrm{kd})$ synthetic compounds that possess high affinity and specificity for their target proteins (equilibrium dissociation constants ranging from $0.05-40 \mathrm{nM}$ ). Thus, they embody the affinity properties of monoclonal antibodies with the chemical production properties of small peptides. In addition, preclinical and clinical studies to date have shown that aptamers and compounds of similar composition are well tolerated, exhibit low or no immunogenicity, and are suitable for repeated administration as therapeutic compounds (EyetechStudyGroup 2002, 2003; Dyke et al., 2006). Moreover, bioavailability and clearance mechanisms of aptamers can be rationally altered by molecular modifications to the ligand (i.e., cholesterol or polyethylene glycol). Most importantly, Rusconi et al. $(2002,2004)$ and Nimjee et al. (2006) have shown that antidote oligonucleotides can be rationally designed that negate the effect of aptamers in vitro and in vivo. Antiplatelet agents currently used in clinics can have a major bleeding side effect which can increase mortality and morbidity and significantly limit their use (Jackson and Schoenwaelder, 2003). Using antidotes is the most effective and reliable way to control drug action and can reduce bleeding associated with current antiplatelet agent use in clinics, enhancing safety and reducing morbidity and mortality.

We used a technique termed convergent SELEX and isolated a number of aptamers that bind to VWF with high affinity. Furthermore, we showed that two of these 
clones inhibit VWF mediated platelet activation and aggregation in ex vivo assays. Coincidentally, we have demonstrated that both of these functional aptamers bind to the same region of VWF involved in platelet aggregation using VWF SPI and SPIII fragments. To test the characteristics of these aptamers in functional assays, we

A

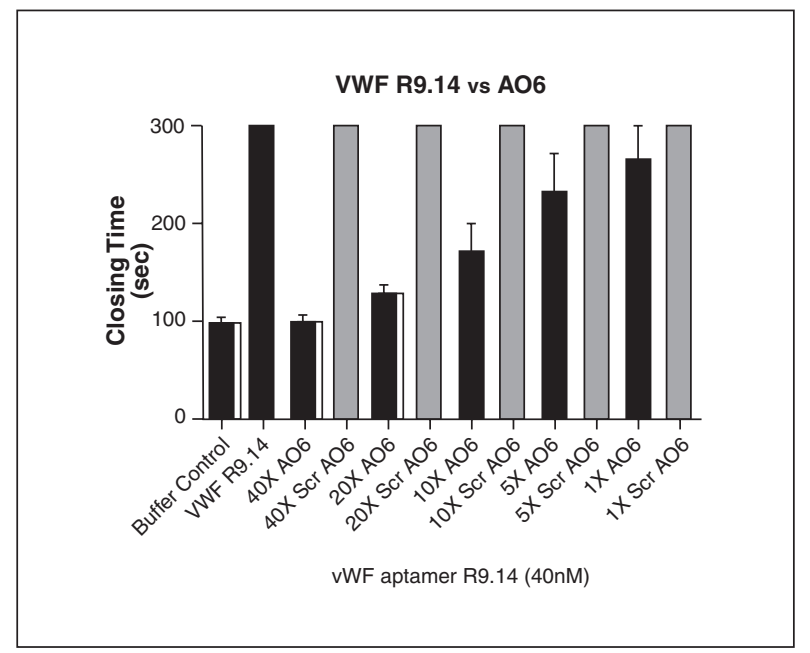

B

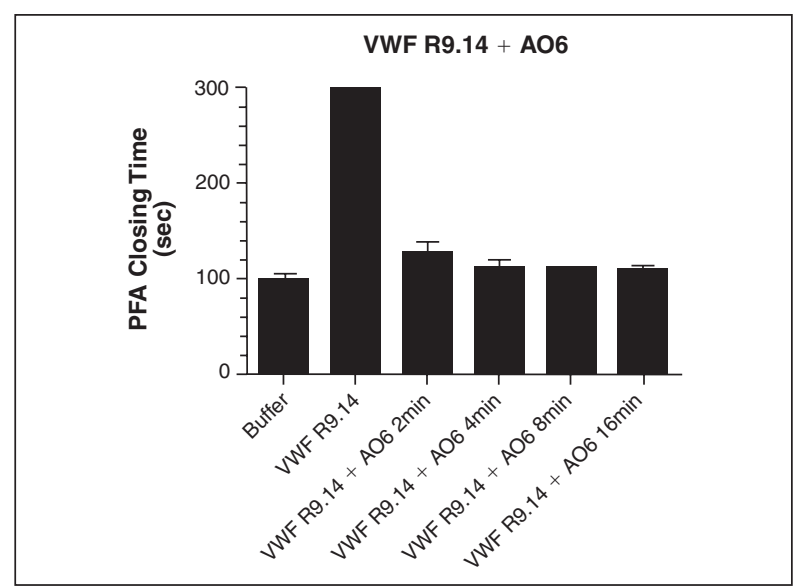

C

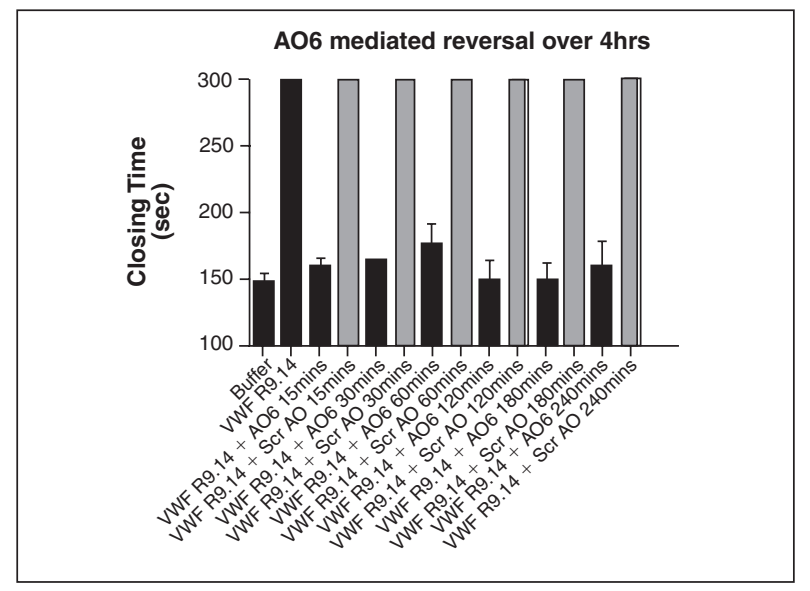

utilized a PFA-100 instrument. PFA-100 simulates platelet function in whole blood under high shear stress and is particularly sensitive to VWF defects (Harrison, 2005). Both clone R9.3 and R9.14 completely inhibited platelet plug formation in PFA-100 at concentrations greater than $40 \mathrm{nM}$ (closing time $>300$ seconds). Moreover, we tested these aptamers in ristocetin, ADP, thrombin (SFLLRN peptide), and collagen-mediated platelet aggregation assays for pathway specificity. Both of these clones inhibited RIPA at greater than $250 \mathrm{nM}$ concentration but had no significant effect in other agonist mediated aggregation assays. These experiments show that both clone R9.3 and clone R9.14 bind VWF with high affinity and inhibit platelet aggregation through inhibition of the GP Ib-IX-V-VWF interaction. This interaction is especially important around areas of high shear stress (i.e., stenosed arteries) and is a valid target for antiplatelet therapy.

Antidote control gives physicians added control over drug activity and provides a safer means for antiplatelet therapy. To further improve the safety of the lead molecule R9.14, we rationally designed an antidote oligonucleotide using the properties inherent to nucleic acids (Rusconi et al. 2002, 2004; Nimjee et al., 2006). Antidote oligonucleotides bind to their target aptamer through Watson-Crick base pairing, thus changing the aptamer's conformational shape and inhibiting binding to its target, therefore reversing its activity. We designed six different antidote oligonucleotides and tested their activity in a nitrocellulose filter binding assay. AO6 was the most effective in inhibiting aptamer binding to VWF, completely reducing it to nonspecific, background levels. To test the effect of antidote AO6 on clone R9.14, we tested the pair in PFA-100. AO6 completely reverses the antiplatelet effect of R9.14 in less than 2 minutes and is effective for at least 4 hours. This aptamer-antidote pair can potentially give physicians a rapid, effective, and continual way to regulate antiplatelet therapy.

FIG. 4. Antidote oligonucleotides to aptamer von Willebrand factor (VWF) R9.14 can reverse aptamer function rapidly and for a prolonged period of time. A: Antidote oligonucleotide 6 (AO6) completely reverses aptamer function in a PFA-100 assay (black bars) at a 40:1 ratio. A scrambled antidote oligonucleotide was used as a negative control (grey bars). Error bars represent the range of data. Each data point was done in triplicate. B: AO6 achieved complete reversal of aptamer VWF R9.14 function in a PFA-100 assay in 2 minutes. AO6 was used at a 40:1 ratio to VWF R9.14 (40 nM). Error bars represent the range of data. Each data point was done in triplicate. C: AO6 inhibits aptamer VWF R9.14 function for 4 hours in a PFA-100 assay (black bars). A scrambled antidote oligonucleotide was used as a negative control (grey bars). Error bars represent the range of data. Each data point was done in triplicate. 


\section{ACKNOWLEDGMENTS}

We would like to thank Dr. J. Evan Sadler for VWF SPI and SPIII fragments and also for helpful discussions. We would like to thank Dr. Jose Lopez for helpful discussions. This work was supported by National Institutes of Health grant HL65222 to B.A.S., a DTRI pilot grant 1UL1RR024128-01 to R.C.B. and B.A.S., and a predoctoral fellowship award from the American Heart Association to S.O.

\section{REFERENCES}

BECKER, R.C., RUSCONI, C., and SULLENGER, B. (2005). Nucleic acid aptamers in therapeutic anticoagulation. Technology, development and clinical application." Thromb. Haemost. 93, 1014-1020.

BHATT, D.L. and TOPOL, E.J. (2003). Scientific and therapeutic advances in antiplatelet therapy. Nat. Rev. Drug Discov 2, 15-28.

CADROY, Y., HANSON, S.R., KELLY, A.B., MARZEC, U.M., EVATT, B.L., KUNICKI, T.J., MONTGOMERY, R.R., and HARKER, L.A. (1994). Relative antithrombotic effects of monoclonal antibodies targeting different platelet glycoprotein-adhesive molecule interactions in nonhuman primates. Blood 83, 3218-3224.

CAUWENBERGHS, N., MEIRING, N., VAUTERIN, S., VAN WYK, V., LAPRECHT, S., ROODT, J.P., NOVAK, L., HARSFALVI, J., DECKMYN, H., and KOTZE, H.F. (2000). Antithrombotic effect of platelet glycoprotein Ibblocking monoclonal antibody Fab fragments in nonhuman primates. Arterioscler. Thromb. Vasc. Biol. 20, 1347-1353.

CHANG, M.C., LIN, H.K., PENG, H.C., and HUANG, T.F. (1998). Antithrombotic effect of crotalin, a platelet membrane glycoprotein $\mathrm{Ib}$ antagonist from venom of Crotalus atrox. Blood 91, 1582-1589.

DYKE, C.K., STEINHUBL, S.R., KLEIMAN, N.D., CANNON, R.O., ABERLE, L.G., LIN, M., MYLES, S.K., MELLONI, C., HARRINGTON, R.A., ALEXANDER, J.H., BECKER, R.C., and RUSCONI, C.P. (2006). First-in-human experience of an antidote-controlled anticoagulant using RNA aptamer technology: a phase 1a pharmacodynamic evaluation of a drug-antidote pair for the controlled regulation of factor IXa activity. Circulation 114, 2490-2497.

EYETECHSTUDYGROUP. (2002). Preclinical and phase 1A clinical evaluation of an anti-VEGF pegylated aptamer (EYE001) for the treatment of exudative agerelated macular degeneration. Retina 22, 143-152.

EYETECHSTUDYGROUP. (2003). Anti-vascular endothelial growth factor therapy for subfoveal choroidal neovascularization secondary to age-related macular degeneration: phase II study results. Ophthalmology 110, 979-986.

FITZWATER, T., and POLISKY, B. (1996). A SELEX primer. Methods Enzymol. 267, 275-301.

HARRISON, P. (2005). Platelet function analysis. Blood Rev. 19, 111-123.

HUYNH, T., THEROUX, P., BOGATY, P., NASMITH, J., and SOLYMOSS, S. (2001). Aspirin, warfarin, or the combi- nation for secondary prevention of coronary events in patients with acute coronary syndromes and prior coronary artery bypass surgery. Circulation 103, 3069-3074.

JACKSON, S.P., and SCHOENWAELDER, S.M. (2003). Antiplatelet therapy: in search of the 'magic bullet'. Nat. Rev. Drug Discov. 2, 775-789.

LAYZER, J. a. S., B.A. (2007). Simultaneous generation of aptamers to multiple gamma-carboxyglutamic acid proteins from a focused aptamer library using DeSELEX and Convergent Selection. Oligonucleotides 17, 1-11.

MASON, P.J., JACOBS, A.K., and FREEDMAN, J.E. (2005). Aspirin resistance and atherothrombotic disease. J. Am. Coll. Cardiol. 46, 986-993.

MILLER, J.L., THIAM-CISSE, M., and DROUET, L.O. (1991). Reduction in thrombus formation by PG-1 F(ab')2, an anti-guinea pig platelet glycoprotein Ib monoclonal antibody. Arterioscler. Thromb. 11, 1231-1236.

NIMJEE, S.M., KEYS, J.R., PITOC, G.A., QUICK, G., RUSCONI, C.P., and SULLENGER, B.A. (2006). A novel antidote-controlled anticoagulant reduces thrombin generation and inflammation and improves cardiac function in cardiopulmonary bypass surgery. Mol. Ther. 14, 408-415.

NIMJEE, S.M., RUSCONI, C.P., HARRINGTON, R.A., and SULLENGER, B.A. (2005a). The potential of aptamers as anticoagulants. Trends Cardiovasc. Med. 15, 41-45.

NIMJEE, S.M., RUSCONI, C.P., and SULLENGER, B.A. (2005b). Aptamers: An emerging class of therapeutics. Annu. Rev. Med. 56, 555-583.

RUGGERI, Z.M. (2003). Von Willebrand factor, platelets and endothelial cell interactions. J. Thromb. Haemost. 1, 1335-1342.

RUGGERI, Z.M., DENT, J.A., and SALDIVAR, E. (1999). Contribution of distinct adhesive interactions to platelet aggregation in flowing blood. Blood 94, 172-178.

RUSCONI, C.P., ROBERTS, J.D., PITOC, G.A., NIMJEE, S.M., WHITE, R.R., QUICK, G., JR., SCARDINO, E., FAY, W.P., and SULLENGER, B.A. (2004). Antidote-mediated control of an anticoagulant aptamer in vivo. Nat. Biotechnol. 22, 1423-1428.

RUSCONI, C.P., SCARDINO, E., LAYZER, J., PITOC, G.A., ORTEL, T.L., MONROE, D., and SULLENGER, B.A. (2002). RNA aptamers as reversible antagonists of coagulation factor IXa. Nature 419, 90-94.

RUSCONI, C.P., YEH, A., LYERLY, H.K., LAWSON, J.H., and SULLENGER, B.A. (2000). Blocking the initiation of coagulation by RNA aptamers to factor VIIa. Thromb. Haemost. 84, 841-848.

SADLER, J.E. (2005). New concepts in von Willebrand disease. Annu. Rev. Med. 56, 173-191.

SADLER, J.E. (2005). von Willebrand factor: two sides of a coin. J. Thromb. Haemost. 3, 1702-1709.

THOM, T., HAASE, N., ROSAMOND, W., HOWARD, V.J., RUMSFELD, J., MANOLIO, T., ZHENG, Z.J., FLEGAL, K., O'DONNELL, C., KITTNER, S., LLOYD-JONES, D., GOFF, D.C., JR., HONG, Y., ADAMS, R., FRIDAY, G., FURIE, K., GORELICK, P., KISSELA, B., MARLER, J., MEIGS, J., ROGER, V., SIDNEY, S., SORLIE, P., STEINBERGER, J., WASERTHIEL-SMOLLER, S., WILSON, M., and WOLF, P. (2006). "Heart disease and stroke statistics-2006 update: a report from the American Heart Associ- 
ation Statistics Committee and Stroke Statistics Subcommittee." Circulation 113, e85-151.

WONG, I.. and LOHMAN, T.M. (1993). A double-filter method for nitrocellulose-filter binding: application to protein-nucleic acid interactions. Proc. Natl. Acad. Sci. USA 90, $5428-5432$.

WU, D., MEIRING, M., KOTZE, H.F., DECKMYN, H., and CAUWENBERGHS, N. (2002a). Inhibition of platelet glycoprotein Ib, glycoprotein IIb/IIIa, or both by monoclonal antibodies prevents arterial thrombosis in baboons. Arterioscler. Thromb. Vasc. Biol. 22, 323-328.

WU, D., VANHOORELBEKE, K., CAUWENBERGHS, N., MEIRING, M., DEPRAETERE, H., KOTZE, H.F., and DECKMYN, H. (2002). Inhibition of the von Willebrand (VWF)-collagen interaction by an antihuman VWF monoclonal antibody results in abolition of in vivo arterial platelet thrombus formation in baboons. Blood 99, 3623-3628.

YAMAMOTO, H., VREYS, I., STASSEN, J.M., YOSHIMOTO, R., VERMYLEN, J., and HOYLAERTS, M.F.
(1998). Antagonism of vWF inhibits both injury induced arterial and venous thrombosis in the hamster. Thromb. Haemost. 79, 202-210.

Address reprint requests to:

Dr. Bruce Sullenger Box 2601

Duke Translational Research Institute

Department of Surgery

University Program in Genetics and Genomics

Duke University Medical Center

Durham, NC 27710

E-mail: bruce.sullenger@duke.edu

Received May 10, 2007; accepted after revision May 24, 2007. 
This article has been cited by:

1. J. L. DIENER, H. A. DANIEL LAGASSÉ, D. DUERSCHMIED, Y. MERHI, J-F. TANGUAY, R. HUTABARAT, J. GILBERT, D. D. WAGNER, R. SCHAUB. 2009. Inhibition of von Willebrand factor-mediated platelet activation and thrombosis by the anti-von Willebrand factor A1-domain aptamer ARC1779. Journal of Thrombosis and Haemostasis 7:7, 1155-1162. [CrossRef]

2. Günter Mayer. 2009. Die chemische Biologie von Aptameren. Angewandte Chemie 121:15, 2710-2727. [CrossRef]

3. Günter Mayer. 2009. The Chemical Biology of Aptamers. Angewandte Chemie International Edition 48:15, 2672-2689. [CrossRef]

4. Yingmiao Liu, Chien-Tsun Kuan, Jing Mi, Xiuwu Zhang, Bryan M. Clary, Darell D. Bigner, Bruce A. Sullenger. 2009. Aptamers selected against the unglycosylated EGFRvIII ectodomain and delivered intracellularly reduce membrane-bound EGFRvIII and induce apoptosis. Biological Chemistry 390:2, 137-144. [CrossRef]

5. Nigel Mackman. 2008. Triggers, targets and treatments for thrombosis. Nature 451:7181, 914-918. [CrossRef] 